CLICdp-Conf-2017-012

30 June 2017

\title{
A multi-chip data acquisition system based on a heterogeneous system-on-chip platform
}

\author{
A. Fiergolski ${ }^{1) *}$ \\ On behalf of the CLICdp collaboration \\ ${ }^{*}$ CERN, Switzerland
}

\begin{abstract}
The Control and Readout Inner tracking BOard (CaRIBOu) is a versatile readout system targeting a multitude of detector prototypes. It profits from the heterogeneous platform of the Zynq System-on-Chip (SoC) and integrates in a monolithic device front-end FPGA resources with a back-end software running on a hard-core ARM-based processor. The userfriendly Linux terminal with the pre-installed DAQ software is combined with the efficiency and throughput of a system fully implemented in the FPGA fabric. The paper presents the design of the SoC-based DAQ system and its building blocks. It also shows examples of the achieved functionality for the CLICpix2 readout ASIC.
\end{abstract}

Talk presented at TIPP conference, Beijing, China, 22-26 May 2017

(C) 2017 CERN for the benefit of the CLICdp Collaboration.

Reproduction of this article or parts of it is allowed as specified in the CC-BY-4.0 license.

${ }^{1}$ Adrian.Fiergolski@cern.ch 


\title{
A multi-chip data acquisition system based on a heterogeneous system-on-chip platform
}

\author{
Adrian Fiergolski \\ on behalf of the CLIC detector and physics (CLICdp) collaboration \\ The European Organization for Nuclear Research, \\ Geneva, Switzerland \\ Adrian.Fiergolski@cern.ch
}

\begin{abstract}
The Control and Readout Inner tracking BOard (CaRIBOu) is a versatile readout system targeting a multitude of detector prototypes. It profits from the heterogeneous platform of the Zynq System-on-Chip (SoC) and integrates in a monolithic device front-end FPGA resources with a back-end software running on a hard-core ARM-based processor. The user-friendly Linux terminal with the pre-installed DAQ software is combined with the efficiency and throughput of a system fully implemented in the FPGA fabric. The paper presents the design of the SoC-based DAQ system and its building blocks. It also shows examples of the achieved functionality for the CLICpix2 readout ASIC.
\end{abstract}

Keywords: electronics, DAQ, SoC, Zynq

\section{Motivation}

The development of pixel detectors for future high-energy physics experiments often implies the use of a custom data acquisition (DAQ) system for a given device. As a consequence, the process of a new chip characterisation often involves an extra effort associated with commissioning and debugging of new hardware, firmware and software of the accompanying DAQ system. Although from a functional point of view the DAQ systems are very similar, different implementations and the lack of cross-compatibility imply some learning stage for the pixel detector user. Moreover, each new DAQ system often requires some integration effort with a test-beam infrastructure. All those aspects delay the pixel detector studies.

The Control and Readout Inner tracking BOard (CaRIBOu) addresses this issue. It is a versatile modular readout system supporting by design a wide range of current and future devices. Integration of new devices requires minimal effort. Since CaRIBOu targets laboratory and high-rate test-beam measurements, the system combines flexibility and high-performance requirements. The project is maintained by a collective effort of a user community. All hardware, firmware and software design files are open source and shared through a public repository [1. 


\section{CaRIBOu}

\subsection{Hardware}

The hardware architecture of the CaRIBOu DAQ system 3. is presented in Figure 1. The detector devices are placed on application specific chipboards. Currently, CaRIBOu supports CLICpix2, C3PD [2, FEI4 [3] and H35Demo [3] chipboards. In addition, there is ongoing work on the integration of the SOICracow 4 device. The chipboards provide minimal functionality like routing between the SEAF 320 Pin connector [5] and the chip, and convenient test points. If needed, the chipboards are equipped with specific buffers, e.g. LVDSCML converters. It makes their design straightforward and cost effective in case of a small volume production.

The chipboard is directly connected to the interface Control and Readout (CaR) board. CaR is a suitable solution for various target chips providing a hardware environment for operating them. The CaR board supports many voltage levels and communication standards, including full-duplex serial links. Moreover, as it is equipped with a set of Analogue-to-Digital Converters (ADCs), the CaR board provides monitoring capabilities, thus enabling measurements without the use of dedicated laboratory equipment. The direct connection with the chipboard allows for all required voltage regulators, ADCs, bias sources and a the clock generator to be placed close to the chip. The full specification of the CaR board is provided elsewhere [6].

The core of the system is a Xilinx Zynq System-on-Chip (SoC) device hosted by the ZC-706 evaluation kit [7. It combines a dual-core ARM Cortex-A9 processor and a Kintex-7 Field Programmable Gate Array (FPGA) fabric connected through a silicon interposer. In order to prevent radiation damage from sources or particle beams and facilitate mounting, the CaRIBOu hardware architecture (Figure 1) enables placement of the evaluation kit at a safe distance from the CaR board through a $\sim 50 \mathrm{~cm}$ long FMC cable. The ZC-706 board supports up to two interface boards. Its commercial applications and evaluation character make it an easily available, cost effective and a rapid solution for small volumes.

In the CaRIBOu application, the ARM processor runs the Linux operating system and the actual DAQ software. The board is accessed through a Secure Shell (ssh) connection (1 Gbps Ethernet) or UART. The processor is efficient

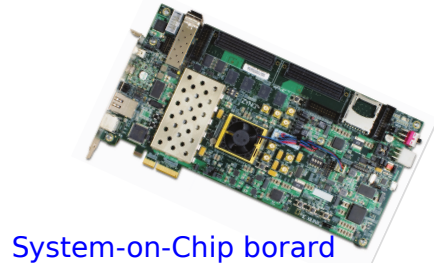
(ZC-706)
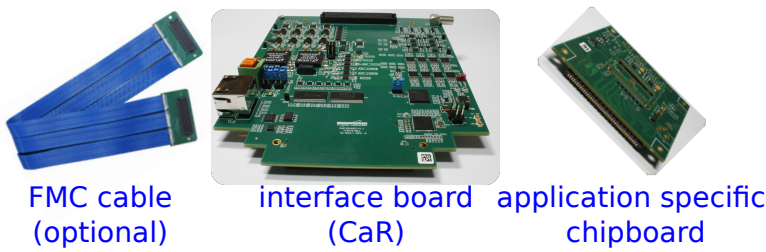

Fig. 1: The hardware architecture of the CaRIBOu DAQ system. 
enough to perform a prompt local data analysis enabling data quality monitoring, calibration, etc. Data are then transferred through 1 Gbps (RJ45 connector). In future versions a $10 \mathrm{Gbps}(\mathrm{SFP}+)$ Ethernet link can be supported. Moreover, the user has the possibility to use other interfaces of the evaluation kit (USB, SD card, PCIe), which are supported by the Linux kernel out of the box.

\subsection{DAQ}

The non-hardware part of the CaRIBOu DAQ consists of 3 components: a DAQ software framework (Peary), a custom Linux distribution (Meta-caribou) and an FPGA processing image (Peary-firmware). Its scheme is presented in Figure 2. The user application gains unified access to hardware using a hardware abstraction layer (HAL) of Peary. The HAL translates its requests to various Linux device driver calls which enable control of different interface controllers and hardware components of the SoC platform (available in the ARM processor periphery or in the FPGA fabric). Data from the application specific chip is transferred through the FPGA fabric to the main memory from where it can be accessed by the processor or pushed further to the Ethernet interface.

The main advantage of Peary is its user-friendly HAL enabling control of the CaR board and the chip. The user can initialize its device with a single command. Using human readable configuration files, the HAL will provide all necessary bias voltages, currents and will set the operational conditions (clock, reset, etc.). The HAL supports the operation of several devices in parallel. Integration of new devices requires minimum code development and is mainly limited to data processing before storing. A device manager of Peary enables dynamic linking of this code based on a device name specified in the configuration files. The Peary framework implements common modules such as a logging engine. It also

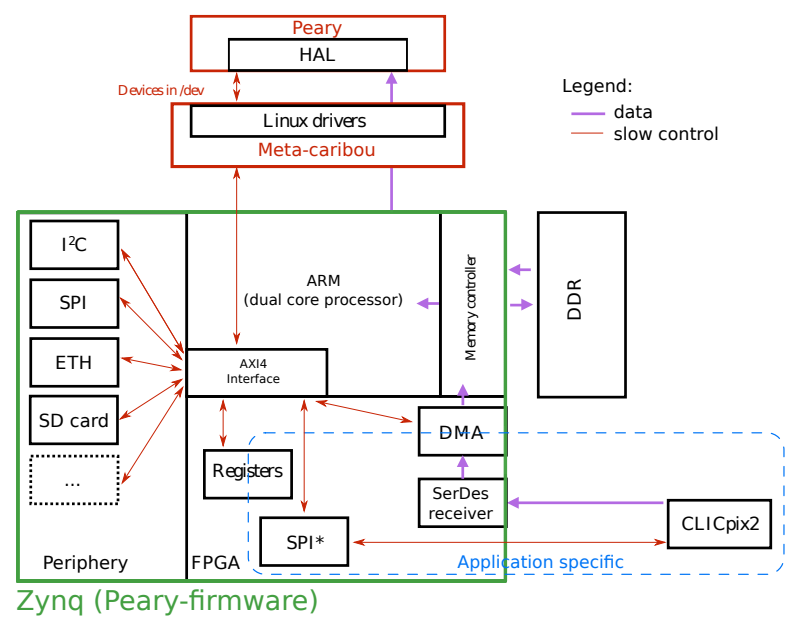

Fig. 2: Scheme of the CaRIBOu DAQ. 
provides a command line interface (CLI) enabling sequential step-by-step control of the chip. This feature proves to be useful at the commissioning stage of a new device. Finally, by supporting DAQ clients, Peary facilitates integration with a top level DAQ run control for combined runs with a test-beam telescope.

In order to facilitate the creation of a custom embedded Linux distribution, CaRIBOu provides a Meta-caribou layer to the Yocto project [10]. Yocto is a Linux build framework supported by a large community of open-source and industrial developers. Meta-caribou customizations set a console-only image with a full-featured Linux system functionality. The image comes with popular packages (python, ssh, gdb, etc.) already pre-installed. Part of Meta-caribou configures a Secondary Program Loader (SPL) which, at the boot stage, loads FPGA firmware (bitfile from Peary-firmware) and sets the ARM CPUs in the desired state. Moreover, the CaRIBOu layer provides the CaR specific hardware description to the Linux kernel through device tree configuration. With a single command, a user launches a build process which fetches the required sources, cross-compiles them and generates an image of the operating system. Further, using a script provided by Meta-caribou, the user prepares an SD card which is eventually inserted into the SD socket of the ZC-706 evaluation kit.

The last component of the CaRIBOu DAQ is the Peary-firmware producing an FPGA image file. It is the only part of the CaRIBOu project utilising proprietary tools (Xilinx Vivado [8]). The Peary-firmware design is handled by the Xilinx IP Integrator [9]. As all autonomous firmware blocks are described according to the IP-XACT standard [11, the tool is aware of their interfaces and can facilitate their integration. Moreover, using the tool, the Peary-firmware handles the configuration of the SoC by defining processor periphery settings, address space and clock frequencies. The user has access to a library of Vivado intellectual property (IP) cores (i.e. DMA, SPI, I2C, etc.) which often come with Linux device drivers distributed through a Yocto layer and maintained by the Xilinx community users. In addition, the Peary-firmware comes with a repository of custom sub-modules (like a serial receiver), which facilitate the development of application-specific blocks providing access to the chip. Some of the sub-modules make use of the System Verilog language supported in Vivado. In case processor interrupts are not required, the custom blocks can be accessed through a generic /dev/mem Linux device driver. The repository contains software examples of this feature. Finally, the Xilinx tools are used by the Peary-firmware also to create the Hardware Description File (HDL) which is required by Meta-caribou for Linux device tree and SPL generation.

\section{Example use case: CLICpix2 readout}

The CaRIBOu DAQ system was used to commission the CLICpix2 readout chip. CLICpix2 is the successor of the CLICpix device [12]. It is produced in a $65 \mathrm{~nm}$ process technology and comes with a larger pixel matrix (128x128) as well as extended Time over Threshold ( 5 bits) and Time of Arrival ( 8 bits) counters in every pixel. The readout protocol of CLICpix2 is based on an Ethernet-like 
$640 \mathrm{Mbps}$ serial stream. The device is configured over the Serial Peripheral Interface (SPI) bus running at $100 \mathrm{MHz}$. The advanced features of CLICpix2, like data compression, frame encoding, test pulsing and power pulsing, put additional requirements on its DAQ system.

The CaRIBOu DAQ system was used in order to obtain preliminary CLICpix2 results. The framework enabled readout of the chip using Zynq serial transceivers and was efficient enough to perform software-based frame decoding and decompression. The chip was properly controlled over the fast SPI protocol. CaRIBOu enabled adjustment and monitoring of the power provided by the CaR board. The card resources were also used to generate clock signals used by the chip. The properly operated Digital-to-Analogue Converters (DAC) of the CaR board allowed to perform bias voltage and current source scans. Moreover, local ADCs enabled voltage measurements without involving external laboratory tools.

\section{Summary}

CaRIBOu integrates a front-end and back-end DAQ system. It is a modular solution targeting laboratory and high-rate test beam measurements. CaRIBOu comes with versatile hardware, firmware and software. The system provides the unique user experience of a regular fully-functional Linux terminal. Due to its heterogeneous nature utilising the Zynq SoC, the framework enables rapid development. The system remains fully flexible leaving the choice of programming language to the user and providing out of the box access to all interfaces supported by the Linux kernel (Ethernet, USB, SD card, etc.). Allowing to focus on the application-specific features, CaRIBOu enables easy integration of new devices. The system was successfully tested during the CLICpix2 commissioning. The open-source nature of the project hosted by a set of user-friendly GitLab repositories enables collaborative system development by a growing developer community.

\section{References}

[1] CaRIBOu project webpage, https://gitlab.cern.ch/Caribou/

[2] I. Kremastiotis, R. Ballabriga, M. Campbell, D. Dannheim, A. Fiergolski, D. Hynds, S. Kulis, I. Peric, "Design and characterisation of a capacitively coupled HVCMOS sensor chip for the CLIC vertex detector", submitted to JINST journal, arXiv:1706.04470

[3] Hongbin Liu and others, "Development of CaRIBOu: a modular readout system for pixel sensor R\&D", in this proceedings

[4] S. Bugiel, R. Dasgupta, S. Glab, M. Idzik, J. Moron, P. J. Kapusta, W. Kucewicz and M. Turala, "Development of SOI pixel detector in Cracow,", SOIPIX 2015, arXiv: 1507.00864

[5] SEAF connector, https://www.samtec.com/products/seaf

[6] Specification of the CaR board, https://gitlab.cern.ch/Caribou/CaribouHW/blob/master/README.md 
[7] Xilinx Zynq-7000 All Programmable SoC ZC-706 Evaluation Kit, https://www.xilinx.com/products/boards-and-kits/ek-z7-zc706-g.html

[8] Vivado Design Suite User Guide: Getting Started,

https://www.xilinx.com/support/documentation/sw_manuals/xilinx2017_1/ug910. vivado-getting-started.pdf

[9] Vivado Design Suite User Guide: Designing IP Subsystems Using IP Integrator https://www.xilinx.com/support/documentation/sw_manuals/xilinx2017_1/ug994. vivado-ip-subsystems.pdf

[10] Yocto Project https://www.yoctoproject.org/

[11] 1685-2014 IEEE Standard for IP-XACT, Standard Structure for Packaging, Integrating, and Reusing IP Within Tool Flows, https://standards.ieee.org/findstds/standard/1685-2014.html

[12] Valerio Pierpaolo, Nascetti Augusto, Ballabriga Rafael, "Electronic Systems for Radiation Detection in Space and High Energy Physics Applications", Sep 2013, CERN-THESIS-2013-156, http://cds.cern.ch/record/1610583 